\title{
Impedance spectroscopy of polymeric light emitting devices based on different poly $(p$-phenylene-vinylene) derivatives
}

\author{
S. Forero-Lenger *, J. Gmeiner, W. Brütting, M. Schwoerer \\ Experimental Physics II, University of Bayreuth, 95440 Bayreuth, Germany
}

\begin{abstract}
Light emitting devices fabricated from different poly ( $p$-phenylene-vinylene) (PPV) derivatives prepared by the precursor route (the unsubstituted homopolymer, a dimethoxy-substituted copolymer and an acetate-substituted copolymer) and devices based on a soluble dialkoxy-substituted homopolymer are compared with respect to their temperature-dependent impedance characteristics. Although similar activation energies of the conductivity in the range $0.4-0.5 \mathrm{eV}$ are found for all polymers, the magnitude of the dielectric loss is significantly different in the polymers indicating a substantial difference in the amount of doping and/or impurities.
\end{abstract}

Keywords: Impedance; Light emitting devices; Poly( $p$-phenylene-vinylene)

\section{Introduction}

During the last 10 years, conjugated polymers like poly( $p$-phenylene-vinylene) (PPV) have been investigated for their use in organic light emitting device (LED) structures. From the point of view of technology, there has been a rapid development enhancing luminescence and lifetime of the LEDs. Nevertheless, the physics of charge transport and luminescence mechanisms are not completely understood yet. Previous work by our group has shown that the unsubstituted homopolymer in ITO/polymer/Al devices (ITO $=$ indium tin oxide) behaves like a doped organic semiconductor. From capacitance-voltage measurements, an acceptor dopant concentration in the order of $10^{17} \mathrm{~cm}^{-3}$ was determined. The occurrence of doping in PPV devices is not unexpected, since in the used precursor route to PPV the conversion process of the deposited precursor polymer film to the conjugated PPV is performed at elevated temperatures directly on the electrode used for hole injection in LEDs. The precursor leaving groups (especially $\mathrm{HCl}$ ) can easily react with the ITO substrate forming $\mathrm{InCl}_{3}$, which in turn can diffuse into the PPV layer and oxidize (dope) the polymer. The chemical reaction at the ITO substrate has been proven by energy-dispersive X-ray

\footnotetext{
${ }^{*}$ Corresponding author.

E-mail address: stefan.forero@uni-bayreuth.de (S. Forero-Lenger).
}

analysis [1] and the subsequent diffusion by secondary ion mass spectrometry (SIMS) [2]. A comparison of different substrate materials has shown that the degree of doping resulting from such chemical reactions depends on the reactivity of the subtrate material [3]. While devices based on the unsubstituted polymer on $\mathrm{Au}$ substrates did not show measurable doping, in devices on ITO, an acceptor dopant concentration of about $10^{17} \mathrm{~cm}^{-3}$ was found. Thus, we could identify the usage of ITO anodes as a major source for doping in PPV devices. As established in recent work on LEDs based on the unsubstituted homopolymer impedance spectroscopy is a powerful tool to study the occurrence of doping in LED structures especially the energy and concentration of doping states as well as their spatial distribution $[4,5]$. In this work, this method was applied on identically fabricated devices based on four different PPV-derivatives.

\section{Experimental}

The investigated devices were fabricated on ITO-coated glass (ITO-thickness 100-200 nm, sheet-resistance 40 $\Omega / \square$ ). On these substrates, three precursor polymers (the unsubstituted homopolymer, a dimethoxy-substituted copolymer, and an acetate-substituted copolymer) and a soluble dialkoxy-substituted homopolymer (see Fig. 1) were deposited with the doctor blade technique [6]. The Al 
a)

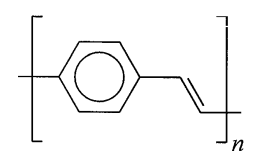
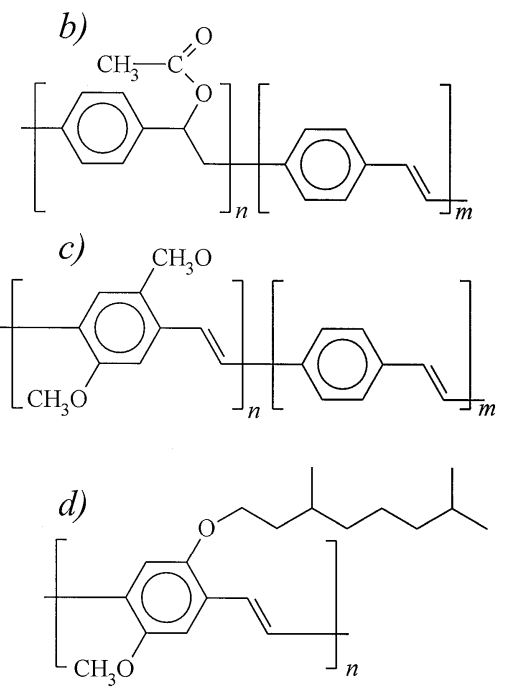

Fig. 1. Investigated PPV derivatives; polymers synthesized by the precursor route: (a) unsubstituted homopolymer, (b) acetate-substituted copolymer $(n \approx 30 \%, m \approx 70 \%)$, (c) dimethoxy-substituted copolymer $(n \approx$ $10 \%, m \approx 90 \%$ ) and a soluble polymer: (d) dialkoxy-substituted homopolymer.

cathodes (about $60 \mathrm{~nm}$ in thickness) were fabricated by thermal evaporation at a pressure ca. $10^{-5}$ mbar. Two consecutive films were deposited and converted in order to separate possible chemical processes at the ITO/polymer interface from the bulk of the polymer. The layer deposited at first is expected to chemically interact with the ITO substrate. After its conversion, it is assumed to act as a buffer layer between ITO and the second layer converted on top. Both polymer films are ca. 100-nm thick each and so the devices have a total thickness of ca. $200 \mathrm{~nm}$. The soluble dialkoxy-substituted homopolymer, assumed not to interact with the substrate because of no thermal process during preparation, was deposited to give $200 \mathrm{~nm}$ thickness, too. The thickness of the ITO and PPV layers were measured with a Dektak surface profilometer. The measurements of dielectric relaxation (or impedance) were made with a broad band impedance setup as described in Ref. [5]. The frequency-sweeps ranged from $10^{7}$ down to $5 \times 10^{-3} \mathrm{~Hz}$ with an oscillator level of $0.1-0.5 \mathrm{~V}$. Additionally, the temperature was varied between room temperature and $213 \mathrm{~K}$. All measurements were performed in a cryostat in inert-atmosphere $\left(\mathrm{N}_{2}\right)$ at normal pressure.

\section{Results and discussion}

In Fig. 2, the real parts of conductivity and dielectric function and the imaginary part of the latter are shown for all four polymers at $T=293 \mathrm{~K}$. The unsubstituted ho- mopolymer and the soluble dialkoxy-substituted homopolymer show a saturation in conductivity for frequencies $<100 \mathrm{~Hz}$ while the acetate-substituted copolymer and the dimethoxy-substituted copolymer further decrease with frequency and may saturate at much lower values $\left(10^{-14}\right.$ $\mathrm{S} / \mathrm{m})$. Apart from the differences in $\operatorname{Re}(\sigma)$ at low frequencies, there are characteristic features seen in the frequency range $10^{2}-10^{4} \mathrm{~Hz}$. They can be better visualized in the imaginary part of $\varepsilon$ since by the relation $\operatorname{Im}(\varepsilon)=$ $\operatorname{Re}(\sigma) /\left(\varepsilon_{0} \omega\right)$, the strong frequency dependence in the conductivity is eliminated. There the unsubstituted ho-

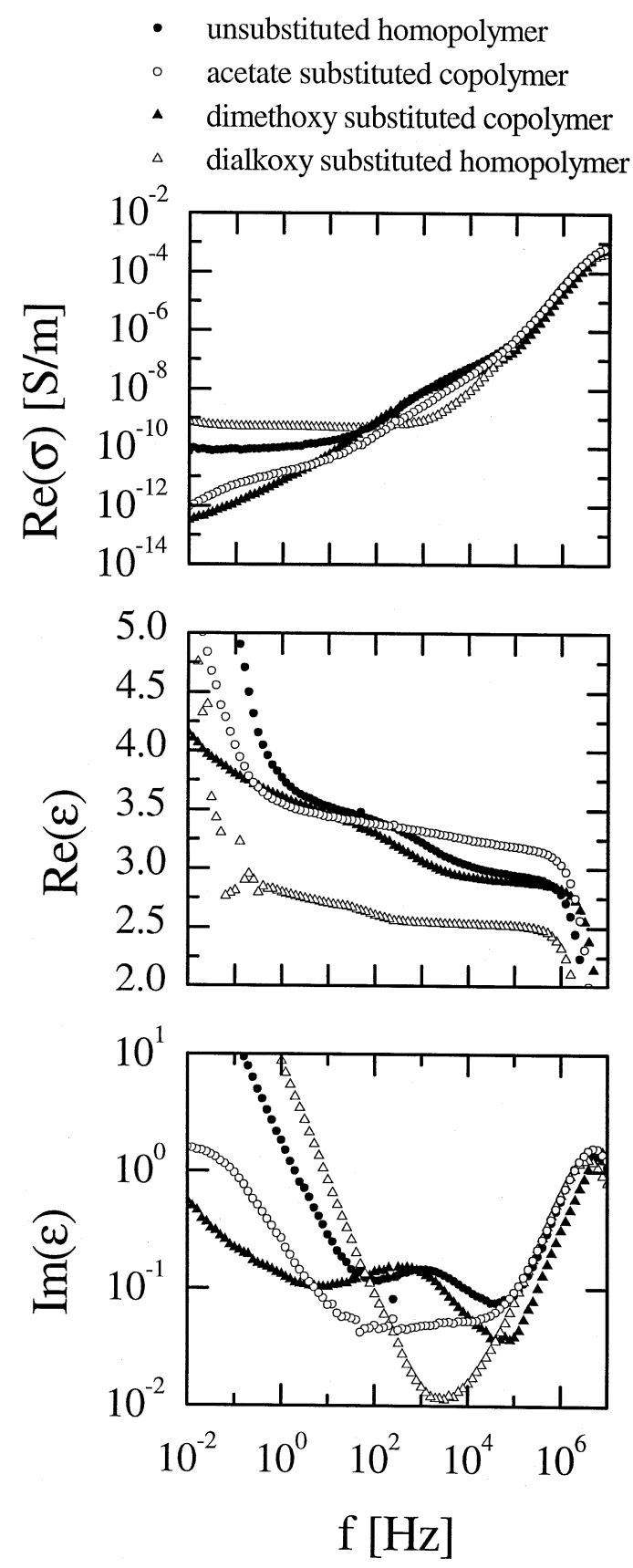

Fig. 2. Real part of the conductivity and real and imaginary parts of the dielectric function at $293 \mathrm{~K}$ for the investigated PPV derivatives. 


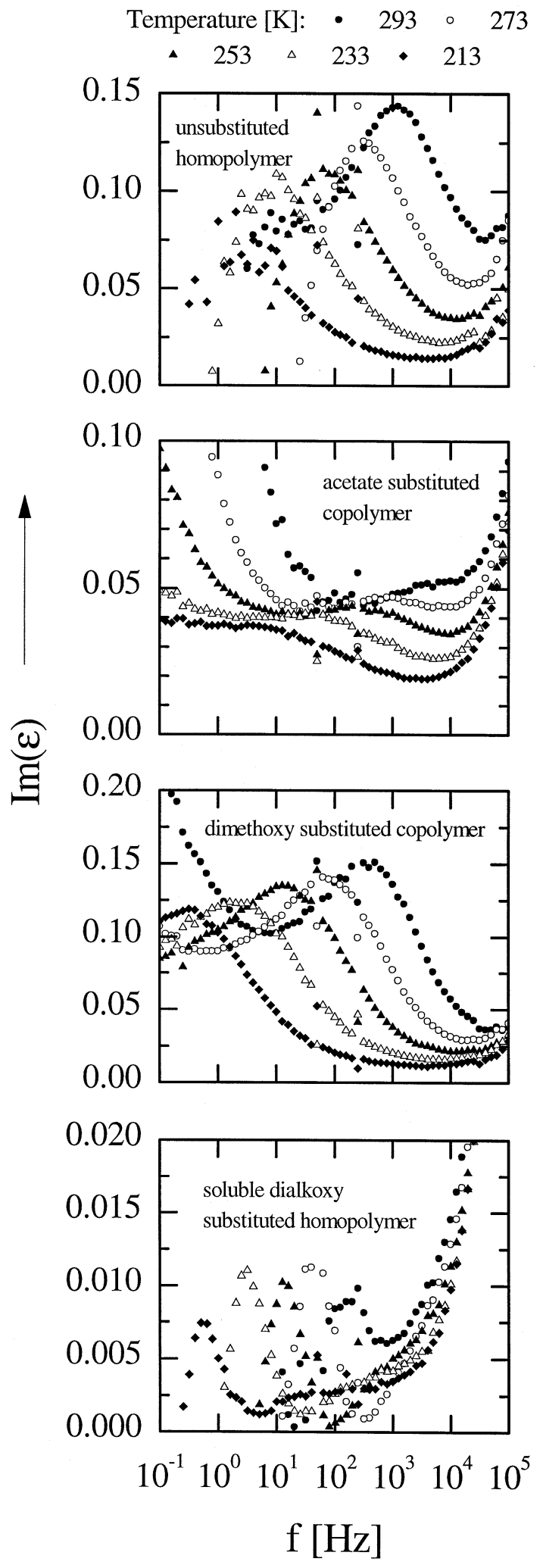

Fig. 3. Imaginary part of the dielectric function (dielectric loss) for different temperatures.

mopolymer and the dimethoxy-substituted copolymer show clearly resolved maxima, which can be related to dielectric loss processes. Values for the acetate-substituted copolymer are about a factor of two lower and the soluble dialkoxy-substituted homopolymer even shows a minimum of the dielectric loss between $10^{3}$ and $10^{4} \mathrm{~Hz}$ with the lowest value of all four polymers. A dielectric loss peak in this frequency range was also observed in a previous work on the unsubstituted homopolymer [5]. There, the high frequency loss peak is interpreted as coming from the bulk conductivity and characterizing the p-doping of the polymer-film with $\mathrm{InCl}_{3}$. A second low frequency loss peak was observed for high thicknesses $(400-1000 \mathrm{~nm})$ and was assigned to the depletion region at the cathode side. In the specimens investigated here, the second polymer layer may be doped to a much lower degree so that the dielectric process connected with the cathode/polymer interface cannot be resolved in the measured frequency window. In order to get further information about the behaviour of the dielectric loss peak due to doping of the polymer bulk material, we have analyzed the temperature dependence of this feature.

Fig. 3 shows the imaginary part of the dielectric function corrected by the contribution of the DC conductivity $\operatorname{Im}(\varepsilon)=\left[\operatorname{Re}(\sigma)-\sigma_{\mathrm{DC}}\right] /\left(\varepsilon_{0} \omega\right)$ in the case of the unsubstituted homopolymer and the soluble dialkoxy-substituted homopolymer. Even the soluble dialkoxy-substituted homopolymer, which is not expected to react with the ITO substrate, shows a dielectric loss peak. Although the magnitude and the visibility of the loss peak is largely different for all four polymers, it is seen that the maximum shifts to lower frequencies with decreasing temperature for all materials. From previous work, it is expected that the temperature dependence of the peak maximum frequencies $f_{0}$ follows a thermally activated behaviour: $f_{0} \propto$ $\exp \left(-E_{a} / k T\right)$.

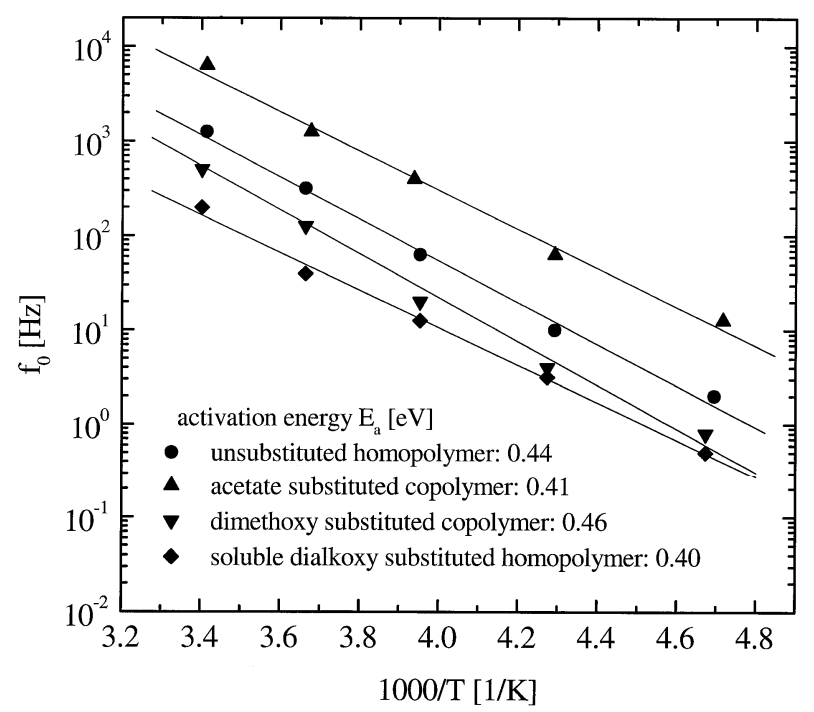

Fig. 4. Arrhenius plot of the temperature-dependent loss peak frequency observed in Fig. 3. 
Fig. 4 shows the temperature dependence of $f_{0}$ in an Arrhenius representation. It can be seen that all four polymers show such a thermally activated behaviour with very similar activation energies between 0.4 and $0.46 \mathrm{eV}$. These values are in good agreement with previous impedance spectroscopy measurements on unsubstituted homopolymer $\left(E_{a}=0.4 \mathrm{eV}\right)$ [5] and also with temperature-dependent mobility measurements on soluble dialkoxy-substituted homopolymer $\left(E_{a}=0.48 \mathrm{eV}\right)$ [7].

\section{Conclusion}

The comparison of temperature-dependent impedance spectra of LEDs based on four different PPV derivatives has shown that in spite of significantly different values of the dielectric loss, the activation energies are very similar in all materials. This result is somewhat unexpected since the soluble PPV is not expected to show a reaction with the ITO substrate. Other possible origins could be a doping by oxygen from the ITO or ambient air or In [8]. Further investigations have to clarify the nature of the observed loss peaks.

\section{Acknowledgements}

We would like to thank Hoechst/Aventis and Cambridge Display Technology for providing some of the materials and the Bayerische Forschungsstiftung and the Sonderforschungsbereich 481 for financial support.

\section{References}

[1] M. Herold, J. Gmeiner, C. Drummer, M. Schwoerer, J. Mater. Sci. 32 (1997) 5709.

[2] G. Sauer, M. Kilo, M. Hund, A. Wokaun, S. Karg, M. Meier, W. Riess, M. Schwoerer, H. Suzuki, J. Simmerer, H. Meyer, D. Haarer, Fresenius' J. Anal. Chem. 353 (1996) 642.

[3] W. Brütting, M. Meier, M. Herold, S. Karg, M. Schwoerer, Chem. Phys. 227 (1998) 243.

[4] A.J. Campbell, D.D.C. Bradley, D.G. Lidzey, J. Appl. Phys. 82 (1997) 326.

[5] J. Scherbel, P.H. Nguyen, G. Paasch, W. Brütting, M. Schwoerer, J. Appl. Phys. 83 (1998) 5045.

[6] M. Herold, J. Gmeiner, M. Schwoerer, Acta Polym. 45 (1994) 392.

[7] P.W.M. Blom, M.J.M. de Jong, M.G. van Munster, Phys. Rev. B 55 (1997) R656.

[8] A.R. Schlatmann, D. Wilms Floet, A. Hilberer, F. Garten, P.J.M. Smulders, Appl. Phys. Lett. 69 (1996) 1764. 